\section{Óbitos infantis evitáveis em Belo Horizonte: análise de concordância da causa básica, 2010-2011}

\section{Avoidable infant deaths in Belo Horizonte: a concordance analysis of basic causes, 2010- 2011}

Simone Passos de Castro e Santos 1

Sônia Lansky 2

Lenice Harumi Ishitani 3

Elisabeth Barboza França 4
1,2 Gerência de Atenção à Saúde. Secretaria Municipal de Saúde de Belo Horizonte. Belo Horizonte, MG, Brasil.

E-mail: sonialansky@gmail.com

3 Gerência de Epidemiologia e Informação. Secretaria Municipal de Saúde de Belo Horizonte.

Belo Horizonte, MG, Brasil.

4 Programa de Pós-Graduação em Saúde Pública. Faculdade de Medicina. Universidade Federal de Minas Gerais. Belo Horizonte, MG, Brasil.

\section{Resumo}

Objetivos: analisar o perfil dos óbitos infantis

Objectives: to examine the profile of avoidable infant deaths investigated and produce a concordance between the basic cause declared on the original death certificate $(D O)$ and the one produced after investigation.

Methods: a population-based study examining infant deaths and avoidable deaths investigated by the Belo Horizonte Death Prevention Committee (CMPOFI), in 2010 and 2011. The death certificate issued after investigation was based on analysis of data from outpatient centers, hospitals and households conducted by the CMPOFI. The causes of death were codified according to the 10th edition of the International Statistical Classification of Diseases and Problems Related to Health and the basic cause selected. The concordance between the basic cause of death on the original death certificate and on the one produced after investigation, analyzed according to the reduced list tabulating the causes of infant mortality (LIR-MI), was determined using the Kappa index.

Results: the Kappa index was weak ( $K=0.389$; CI95\%: 0.192-5.76) when evaluated using the LIR-MI groups. There were significant changes in the cause of death after investigation, with an increase in the proportion of deaths from asphyxia, maternal factors, child infections, perinatal infections, external causes and sudden infant death syndrome.

Conclusions: the investigation of deaths shed greater light on the circumstances of avoidable infant deaths and the classification of the basic cause, which are fundamental steps in guiding action to prevent such deaths.

Key words Cause of death, Infant mortality, Reproducibility of results evitáveis investigados e a concordância entre a causa básica da declaração de óbito (DO) original e da DO após investigação.

Métodos: estudo de base populacional com análise dos óbitos infantis e dos óbitos evitáveis investigados pelo Comitê de Prevenção de Óbitos de Belo Horizonte (CMPOFI), em 2010 e 2011. A DO após investigação baseou-se na análise dos dados ambulatorial, hospitalar e domiciliar realizada pelo segundo a Classificação Estatística Internacional de Doenças e Problemas Relacionados à Saúde - $10^{\circ}$ Revisão e a causa básica selecionada. A concordância entre a causa básica da DO original e da DO após investigação, analisada de acordo com lista reduzida de tabulação de causas de mortalidade infantil (LIR-MI), foi determinada pelo índice Kappa.

Resultados: o Kappa foi fraco (K=0,389; IC95\%: 0,192-05,76) quando avaliado pelos grupos da LIRMI. Houve mudanças relevantes na causa de óbito após investigação, com aumento da proporção de óbitos por asfixia, fatores maternos, infecções da criança, infecções perinatais, causas externas e morte súbita na infância.

Conclusões: a investigação de óbitos possibilitou maior esclarecimento sobre as circunstâncias dos óbitos infantis evitáveis e a qualificação da causa básica, passos fundamentais para orientar as ações para sua prevenção.

Palavras-chave Causas de morte, Mortalidade infantil, Reprodutibilidade dos testes CMPOFI. As causas de morte foram codificadas 


\section{Introdução}

O coeficiente de mortalidade infantil é importante indicador das condições de saúde da população, muito utilizado no planejamento e avaliação das políticas de saúde. 1 O componente neonatal da mortalidade infantil concentra a maior parcela dos óbitos infantis e está associado às condições de assistência ao pré-natal e ao parto. ${ }^{2-4}$ A relevância de se estudar as circunstâncias dos óbitos infantis devese ao fato de serem mortes precoces e potencialmente evitáveis pelo acesso em tempo oportuno a serviços qualificados de saúde. 2,3

Em 2000, na Conferência Mundial das Organizações das Nações Unidas, foram estabelecidos oito Objetivos de Desenvolvimento do Milênio (ODM) com o compromisso de alcance de metas até o ano de 2015. Reduzir em $2 / 3$ a mortalidade na infância é um dos ODM e o Brasil alcançou esta meta antes do prazo, com a redução da mortalidade infantil de 47,1 para 15,3 por mil nascidos vivos (NV) 4 entre 1990 e 2011. No entanto, persistem os diferenciais sociais, econômicos e de acesso a serviços, que precisam ser reconhecidos como determinantes da mortalidade infantil para a diminuição das iniquidades e das injustiças sociais. ${ }^{4,5}$

Em Belo Horizonte, foi significativa a queda da mortalidade infantil nas últimas décadas. No período de 1993 a 2011 a redução foi de $71,6 \%$, passando de 36,6 óbitos por mil NV para 10,4 por $1000 \mathrm{NV}$. A melhoria das condições de vida da população, a ampliação do acesso aos serviços de saúde e às tecnologias disponíveis são fatores que contribuíram para esta considerável redução.1,2,3,6 Por ser mais sensível à ação destes fatores, a queda foi mais importante no componente pós-neonatal no período e, desde 1990, a mortalidade neonatal é o principal componente em termos proporcionais, $1-3,6$ respondendo por $63,5 \%$ da mortalidade infantil em 2011.

Um aspecto relevante e ainda um desafio é a qualificação dos dados e da informação do Sistema de Informação sobre Mortalidade (SIM), principalmente em relação ao correto preenchimento da sequência de causas do óbito na Declaração de Óbito (DO). Para a construção do perfil de mortalidade baseado na causa básica do óbito (CB) é fundamental o monitoramento da qualidade da informação médica nas $\mathrm{DO},{ }^{7}$ necessárias para a adequada seleção da CB. Estudos realizados em áreas metropolitanas do Brasil apontam precariedade na qualidade do preenchimento da DO no tocante à falta de informações e distorções no preenchimento das causas do óbito. ${ }^{8-10}$ As informações geradas a partir dos dados do SIM devem ser consistentes, uma vez que são fundamentais para a programação de ações da saúde 9 e de outras políticas públicas.

Em Belo Horizonte, em 2002 foi constituído o Comitê Municipal de Prevenção de Óbitos Fetal e Infantil BH-Vida/SMSA (CMPOFI) com o objetivo de investigar os óbitos infantis e fetais potencialmente evitáveis para qualificar as informações produzidas na DO e conhecer a magnitude dos problemas relacionados a estes. ${ }^{5}$ O CMPOFI busca construir a história do processo saúde-doença até o momento do óbito, por meio da avaliação de prontuários da assistência na atenção básica, na urgência e no hospital, além de entrevista domiciliar, que fornece informações sobre as condições sociais da família, o percurso na assistência de saúde e a percepção sobre o óbito. O propósito do Comitê é dar visibilidade às questões relacionadas à assistência de saúde e possibilitar a proposição de medidas que possam apoiar as ações de redução da mortalidade infantil, 5 constituindo-se, portanto, como instrumento de gestão. A investigação possibilita produzir informações mais esclarecedoras para além da análise das causas de óbitos baseada apenas nos registros da $\mathrm{DO} 10$ e disponibiliza informações que qualificam o banco de dados do SIM e do Sistema de Informação sobre Nascidos Vivos (SINASC). 11 Nesse contexto este estudo teve como objetivo analisar o perfil dos óbitos infantis evitáveis e avaliar a concordância entre a causa básica da DO original e a causa básica proposta, após investigação realizada pelo CMPOFI.

\section{Métodos}

Trata-se de estudo de base populacional com análise de dados de óbitos infantis ocorridos nos anos de 2010 e 2011, de crianças residentes em Belo Horizonte analisados pelo CMPOFI. Foi definido como óbito infantil evitável aquele com peso ao nascimento maior ou igual a $1500 \mathrm{~g}$ e sem malformação congênita grave (malformação complexa com prognóstico reservado), levando em consideração a viabilidade fetal e o potencial de prevenção. 1-3

Os dados foram obtidos da DO, do SIM, da Declaração de Nascido Vivo (DNV), do SINASC e da investigação (domiciliar, ambulatorial e hospitalar) feita pelo CMPOFI. As informações sobre o peso ao nascimento e a presença de malformação congênita grave foram resgatadas da DO, da DNV e da investigação em prontuário ambulatorial, hospitalar e domiciliar. Foram excluídos do estudo os casos que não havia peso ao nascimento em nenhuma fonte de informação.

A investigação dos casos pelo CMPOFI foi rea- 
lizada por profissionais de nível superior dos Comitês Distritais que utilizaram dados de prontuários da atenção ambulatorial e hospitalar e realizaram entrevista domiciliar com a mãe ou pessoa da família apta a prestar informações. Em todas as etapas da investigação foram utilizados formulários padronizados pelo Ministério da Saúde. 12

Para este estudo foi preenchida uma nova DO (DO após investigação) para cada óbito, de acordo com dados dos formulários de investigação, sem conhecimento prévio da DO original. Na DO após investigação foram consideradas como causas de óbito aquelas registradas por médico e baseada em informações contidas na investigação realizada pelo CMPOFI. Essa etapa foi realizada por dois médicos diferentes e no caso de discordância entre as declarações foi realizada revisão do caso e rediscussão para decisão da sequência de causas na DO após investigação. Para o preenchimento da DO após investigação foram utilizadas as definições descritas no Manual de Instruções de Preenchimento da Declaração de Óbito 201113 e na CID-10.14 Dados disponíveis no SINASC foram utilizados para complementar as informações na DO após investigação, quando necessário.

A codificação das causas de morte na parte I e na parte II da DO após investigação foi realizada segundo as regras da CID-10.14 Essa etapa foi executada por médico treinado e com experiência em codificação das causas de mortalidade. Para o processo de seleção da causa básica da DO após investigação foi utilizado o Sistema de Seleção de Causa Básica.

O perfil dos óbitos infantis investigados pelo CMPOFI foi analisado e comparado com o perfil do total de óbitos notificados no SIM, considerando os componentes da mortalidade infantil e, variáveis relacionadas à assistência: local de ocorrência do óbito (hospital, outros estabelecimentos de saúde, domicílio/via pública) e tipo de parto (vaginal e cesariana); à mãe: idade (menor de 20 anos, 20 a 34 anos e 35 anos ou mais), escolaridade (menos de oito anos de estudo e oito anos ou mais) e paridade (gravidez única e múltipla); e ao nascido vivo: peso ao nascer (menor que $2500 \mathrm{~g}$ e maior ou igual a $2500 \mathrm{~g}$ ), raça/cor (branco e negro), sexo (masculino e feminino) e idade gestacional (menor que 37 semanas e maior ou igual à 37 semanas). Foi realizada a análise da diferença entre proporções nos dois grupos por meio do teste qui-quadrado de Pearson, considerando o nível de significância de $5 \%$.

A análise de concordância entre a causa básica da DO original do banco do SIM e da DO após investigação foi realizada utilizando-se a concordância observada e a ajustada pelo índice Kappa ( $<0,4$ fraca; 0,40-0,75 intermediária e $>0,75$ boa) 15 e o respectivo intervalo de confiança de $95 \%$ (IC95\%). Considerou-se a classificação das causas de acordo com Lista reduzida de tabulação de causas de mortalidade infantil (LIR-MI). ${ }^{16}$

Foi construído banco de dados específico para processamento e análise dos dados utilizando o Microsoft Office Excel. Para as análises estatísticas foram utilizados os software R, versão 3.0.2, e IBM SPSS Statistics versão 20.

Este estudo foi aprovado pelo Comitê de Ética em pesquisa da Universidade Federal de Minas Gerais - Parecer 325.355.

\section{Resultados}

Em 2010 e 2011 foram notificados no SIM 665 óbitos infantis de crianças residentes no município de Belo Horizonte, sendo que, 151(22,7\%) eram óbitos de crianças com peso ao nascer maior ou igual a $1500 \mathrm{~g}$ e sem malformação congênita grave. Dois destes casos $(1,3 \%)$ não foram investigados e considerados como perda, sendo de 149 óbitos a população final deste estudo.

A Tabela 1 apresenta a distribuição dos 665 óbitos infantis notificados ao SIM e dos 149 óbitos investigados segundo variáveis selecionadas.

Nos dois grupos predominaram óbitos ocorridos em hospitais (93,5\% e 75,8\%, respectivamente), crianças do sexo masculino $(55,9 \%$ e $58,4 \%)$, negras $(58,1 \%$ e $61,1 \%)$, nascidas de gravidez única $(89,7 \%$ e $95,3 \%)$, de parto vaginal $(50,8 \%$ e $55,7 \%)$, de mães com oito ou mais anos de estudo $(71,4 \%$ e $61,6 \%)$ e com idade entre 20 e 34 anos $(63,8 \%$ e $65,1 \%)$.

Dentre o total de óbitos infantis houve predomínio do componente neonatal precoce $(50,7 \%)$, de crianças com baixo peso ao nascer $(72,8 \%)$ e prematuras $(70,7 \%)$. No grupo de óbitos investigados prevaleceu o componente pós-neonatal $(54,4 \%)$, crianças com peso adequado ao nascer $(70,5 \%)$ e a termo $(74,1 \%)$. A diferença entre essas variáveis foi estatisticamente significativa.

A escolaridade materna e idade gestacional foram registradas como ignoradas em $12,2 \%$ e $15,8 \%$ das declarações de óbito infantil, respectivamente (Tabela 2).

A causa básica dos óbitos infantis notificados no SIM e a CB dos óbitos infantis evitáveis segundo a DO original e a DO após investigação foram agrupadas de acordo com a LIR-MI e são apresentadas na Tabela 2. As causas relacionadas ao período peri- 
natal (fatores maternos, prematuridade, asfixia/hipóxia, infecções perinatais, afecções respiratórias perinatais e transtornos cardiovasculares originados no período perinatal) predominaram entre os óbitos infantis notificados no $\operatorname{SIM}(53,5 \%)$ e entre os óbitos evitáveis na DO após investigação $(55,1 \%)$. O mesmo não ocorreu em relação aos óbitos evitáveis na DO original, onde somente $40,4 \%$ eram causas relacionadas ao período perinatal. Entre os óbitos infantis notificados no SIM a prematuridade foi a principal causa de óbito, seguida pelas malformações congênitas, infecções perinatais, asfixia/hipóxia, causas externas e fatores maternos. De acordo com a DO original dos óbitos evitáveis, os principais grupos de causa segundo a LIR-MI foram: causas externas, asfixia/hipóxia, infecções perinatais, infecções das crianças, prematuridade, malformações congênitas e os fatores maternos.

$\mathrm{Na}$ DO após investigação predominaram os grupos da asfixia/hipóxia, fatores maternos, infecções da criança, infecções perinatais, causas externas a Síndrome da Morte Súbita na Infância (SMSI) e a prematuridade.

\section{Tabela 1}

Distribuição dos óbitos infantis notificados ao Sistema de Informações sobre Mortalidade e óbitos investigados segundo variáveis selecionadas. Belo Horizonte, 2010 e 2011.

\begin{tabular}{|c|c|c|c|c|c|}
\hline \multirow[t]{2}{*}{ Variável } & \multicolumn{2}{|c|}{ Óbitos infantis } & \multicolumn{2}{|c|}{ Óbitos investigados } & \multirow[t]{2}{*}{$p$} \\
\hline & $\mathrm{n}$ & $\%$ & $\mathrm{n}$ & $\%$ & \\
\hline Componente óbito & & & & & $<0.001$ * \\
\hline Neonatal precoce & 336 & 50,7 & 41 & 27,5 & \\
\hline Neonatal tardio & 114 & 17,2 & 27 & 18,1 & \\
\hline Pós-neonatal & 213 & 32,1 & 81 & 54,4 & \\
\hline Sexo & & & & & 0,581 \\
\hline Feminino & 291 & 44,1 & 62 & 41,6 & \\
\hline Masculino & 369 & 55,9 & 87 & 58,4 & \\
\hline Raça/Cor & & & & & 0,509 \\
\hline Branco & 266 & 41,9 & 58 & 38,9 & \\
\hline Negro & 369 & 58,1 & 91 & 61,1 & \\
\hline Idade da mãe (anos) & & & & & 0,774 \\
\hline$<20$ & 111 & 18,3 & 29 & 19,5 & \\
\hline 20 a 34 & 386 & 63,8 & 97 & 65,1 & \\
\hline 35 ou mais & 108 & 17,9 & 23 & 15,4 & \\
\hline Escolaridade mãe (anos) & & & & & $0.022 *$ \\
\hline$<8$ & 167 & 28,6 & 56 & 38,4 & \\
\hline$\geq 8$ & 417 & 71,4 & 90 & 61,6 & \\
\hline Gravidez & & & & & $0.035 *$ \\
\hline Única & 542 & 89,7 & 142 & 95,3 & \\
\hline Múltipla & 62 & 10,3 & 7 & 4,7 & \\
\hline Tipo de parto & & & & & 0,287 \\
\hline Vaginal & 307 & 50,8 & 83 & 55,7 & \\
\hline Cesária & 297 & 49,2 & 66 & 44,3 & \\
\hline Idade gestacional (semanas) & & & & & $<0.001$ * \\
\hline$<37$ & 396 & 70,7 & 38 & 25,9 & \\
\hline$\geq 37$ & 164 & 29,3 & 109 & 74,1 & \\
\hline Peso ao nascer $(\mathrm{g})$ & & & & & $<0.001 *$ \\
\hline$<2500$ & 446 & 72,8 & 44 & 29,5 & \\
\hline$\geq 2500$ & 167 & 27,2 & 105 & 70,5 & \\
\hline Local de ocorrência & & & & & $<0.001$ * \\
\hline Hospital & 619 & 93,5 & 113 & 75,8 & \\
\hline Outro estabelecimento de saúde & 22 & 3,3 & 14 & 9,4 & \\
\hline Domicílio/Via pública & 21 & 3,2 & 22 & 14,8 & \\
\hline
\end{tabular}

*Teste de qui-quadrado de Pearson significativo a $5 \%$.

Excluídos os dados ignorados

Fonte: SIM e CPOMFI - Secretaria Municipal de Saúde de Belo Horizonte. 
Distribuição proporcional das causas básicas segundo grupamentos da LIR-MI dos óbitos infantis notificados ao Sistema de informações sobre Mortalidade e dos óbitos investigados, segundo a DO original e DO após investigação. Belo Horizonte, 2010 e 2011.

\begin{tabular}{|c|c|c|c|c|c|c|}
\hline \multirow[t]{3}{*}{ LIR-MI* } & \multicolumn{2}{|c|}{$\begin{array}{l}\text { Óbitos infantis } \\
\text { notificados }\end{array}$} & \multicolumn{4}{|c|}{ Óbitos infantis investigados } \\
\hline & \multirow[b]{2}{*}{$\mathrm{n}$} & \multirow[b]{2}{*}{$\%$} & \multicolumn{2}{|c|}{ DO original } & \multicolumn{2}{|c|}{ DO após investigação } \\
\hline & & & $\mathrm{n}$ & $\%$ & $\mathrm{n}$ & $\%$ \\
\hline Fatores maternos & 37 & 5,6 & 2 & 1,3 & 18 & 12,1 \\
\hline Prematuridade & 182 & 27,4 & 5 & 3,4 & 8 & 5,4 \\
\hline Asfixia/Hipóxia & 46 & 6,9 & 29 & 19,5 & 36 & 24,2 \\
\hline Infecções perinatais & 83 & 12,5 & 22 & 14,8 & 17 & 11,4 \\
\hline Afecções respiratórias perinatais & 2 & 0,3 & 1 & 0,7 & 2 & 1,3 \\
\hline \multicolumn{7}{|l|}{ Transtornos cardiovasculares originados } \\
\hline no período perinatal & 5 & 0,8 & 1 & 0,7 & 1 & 0,7 \\
\hline Infecções da criança & 33 & 5,0 & 16 & 10,7 & 18 & 12,1 \\
\hline Desnutrição e anemias nutricionais & 1 & 0,2 & 0 & 0,0 & 1 & 0,7 \\
\hline Asma & 2 & 0,3 & 0 & 0,0 & 2 & 1,3 \\
\hline Causas externas nas crianças & 45 & 6,8 & 36 & 24,2 & 15 & 10,1 \\
\hline Malformações congênitas** & 147 & 22,1 & 4 & $2,7 *$ & 1 & 0,7 \\
\hline Doenças imunizáveis & 1 & 0,2 & 1 & 0,7 & 1 & 0,7 \\
\hline Síndrome da morte súbita na infância & 3 & 0,5 & 1 & 0,7 & 10 & 6,7 \\
\hline Outras causas & 78 & 11,7 & 31 & 20,8 & 19 & 12,8 \\
\hline Total & 665 & 100,0 & 149 & 100,0 & 149 & 100,0 \\
\hline
\end{tabular}

Fonte: SIM e CPOMFI - Secretaria Municipal de Saúde de Belo Horizonte.

*LIR-MI = Lista reduzida de tabulação de causas de mortalidade infantil; DO= Declaração de óbito; **Malformação congênita grave com critério de exclusão para investigação.

A Tabela 3 apresenta a concordância entre a causa básica da DO original e após investigação segundo categorias de causas de mortalidade infantil dos 149 óbitos estudados de acordo com os grupos da LIR-MI. Considerando a DO original, 118 óbitos (79,2\%) foram classificados em algum dos 13 grupos de causas especificadas da LIR-MI e 31 óbitos $(20,8 \%)$ no grupo "outras causas" da LIR -MI. Este grupamento corresponde às causas de óbito que não se enquadraram em nenhum dos grupos específicos da LIR-MI, constituído por causas mal definidas de óbito do capítulo específico da CID (capítulo XVIII, exceto R95), e outros códigos que não caracterizam a causa da morte. 16

Na DO original sete casos $(22,6 \%)$ tiveram a CB "Outros Transtornos Originados no Período Perinatal" (P90-P95), três paralisia cerebral não especificada (G80.9), dois hidrocefalia não especificada, outros três lesão encefálica anóxica (G93.1), que exclui anóxia perinatal, e cinco tinham causa desconhecida (R99). Na DO após investigação, 130 $(87,2 \%)$ óbitos foram classificados nos grupos da LIR-MI, 11 se mantiveram como "outras causas" e oito entraram neste grupo, totalizando $19(12,8 \%)$ óbitos. Sendo assim, 20 casos com causa básica na DO original classificados como "outras causas" na DO após investigação foram classificados em algum dos 13 grupos especificados da LIR-MI. Foram originalmente classificadas como causa externa $36 \mathrm{DO}$, sendo que para $33(91,7 \%)$ houve realização de necropsia; 13 (36,1\%) óbitos ocorreram em hospital, oito $(22,2 \%)$ em Unidade de Pronto Atendimento e $15(41,7 \%)$ no domicílio. Em 31 óbitos $(86,1 \%)$ a causa básica foi classificada como "Fatos e eventos não especificados e intenção não determinada" (Y34), que são causas externas não específicas. Os cinco casos identificados como causa externa após investigação foram: reação vacinal, aspiração de conteúdo gástrico em criança com hidrocefalia, choque anestésico em paciente com malformação cardíaca (sem gravidade), inalação e ingestão de alimento causando obstrução do trato respiratório em criança com lesão encefálica anóxica.

A Tabela 4 apresenta a causa básica de óbito de acordo com a LIR-MI, a concordância observada, o índice Kappa e o IC95\% para a DO original e a DO 


\section{DO após investigação}

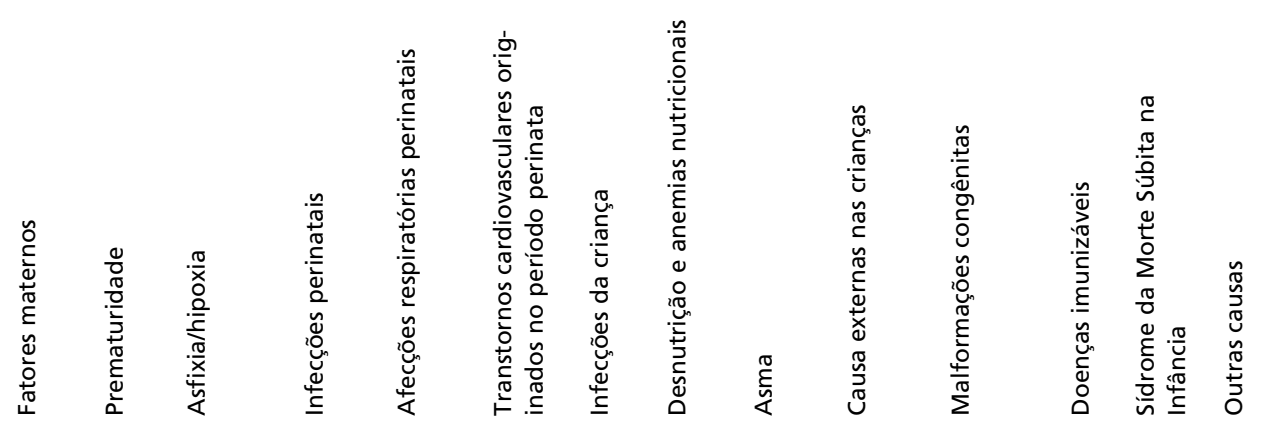

\begin{tabular}{|c|c|c|c|c|c|c|c|c|c|c|c|c|c|c|c|c|}
\hline \multirow{20}{*}{ 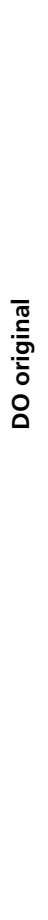 } & Fatores Maternos & 1 & - & - & - & - & 1 & - & - & - & - & - & - & - & - & 2 \\
\hline & Prematuridade & - & 4 & - & - & - & - & - & - & - & - & 1 & - & - & - & 5 \\
\hline & Asfixia/hipoxia & 3 & - & 24 & 1 & - & - & - & - & - & - & - & - & - & 1 & 29 \\
\hline & Infecções perinatais & 5 & 2 & 2 & 10 & 1 & - & - & - & - & - & - & - & 1 & 1 & 22 \\
\hline & Afecções respirató- & & & & & & & & & & & & & & & \\
\hline & rias perinatais & - & - & - & - & 0 & - & 1 & - & - & - & - & - & - & - & 1 \\
\hline & $\begin{array}{l}\text { Transtornos cardio- } \\
\text { vasculares origina- } \\
\text { dos no período }\end{array}$ & & & & & & & & & & & & & & & \\
\hline & perinatal & - & - & 1 & - & - & 0 & - & - & - & - & - & - & - & - & 1 \\
\hline & Infecções da criança & - & - & 2 & 3 & - & - & 9 & - & 1 & 1 & - & - & - & - & 16 \\
\hline & $\begin{array}{l}\text { Desnutrição e ane- } \\
\text { mias nutricionais }\end{array}$ & - & - & - & - & - & - & - & 0 & - & - & - & - & - & - & 0 \\
\hline & Asma & - & - & - & - & - & - & - & - & 0 & - & - & - & - & - & 0 \\
\hline & Causa externas nas & & & & & & & & & & & & & & & \\
\hline & crianças & 2 & 2 & - & 2 & - & - & 6 & - & 1 & 10 & - & - & 7 & 6 & 36 \\
\hline & Malformaçõe & & & & & & & & & & & & & & & \\
\hline & congênitas & 1 & - & 1 & - & - & - & - & 1 & - & 1 & 0 & - & - & - & 4 \\
\hline & Doenças imunizáveis & - & - & - & - & - & - & - & - & - & - & - & 1 & - & - & 1 \\
\hline & Sídrome da morte & & & & & & & & & & & & & & & \\
\hline & súbita na infância & - & - & 1 & - & - & - & - & - & - & - & - & - & 0 & - & 1 \\
\hline & Outras causas & 6 & - & 5 & 1 & 1 & - & 2 & - & - & 3 & - & - & 2 & 11 & 31 \\
\hline & Total & 18 & 8 & 36 & 17 & 2 & 1 & 18 & 1 & 2 & 15 & 1 & 1 & 10 & 19 & 149 \\
\hline
\end{tabular}

Fonte: SIM e CPOMFI - Secretaria Municipal de Saúde de Belo Horizonte.

DO= Declaração de óbito.

após investigação.

O índice Kappa foi fraco para os grupos dos fatores maternos ( $\mathrm{K}=0,08$; IC95\%: 0,091-0,246), asma e para as outras causas de óbito $(\mathrm{K}=0,33$; IC95\%: 0,146-0,524 ); entre intermediário a bom para a prematuridade $(\mathrm{K}=0,6$; IC95\%: 0,279-0,918), asfixia/hipóxia ( $\mathrm{K}=0,67$; IC95\%: 0,521-0,812 ), infecções perinatais ( $\mathrm{K}=0,44$; IC95\%: 0,231-0,650), infecções da criança ( $\mathrm{K}=0,47$; IC95\%: 0,248-0,690), causas externas nas crianças $(\mathrm{K}=0,41$; IC95\%: 0,211-0,615 ) e excelente para as doenças imunizáveis ( $\mathrm{K}=1,0$; IC95\%: 1,0-1,0 ). O índice Kappa e a concordância observada entre a causa básica da DO original e da DO após investigação nos óbitos investigados foi fraco para os grupamentos da LIR-MI $\quad(\mathrm{K}=0,389$; IC95\%：0,192-0,576；CO: $47,0 \%$ ). Verificou-se aumento proporcional importante na DO após investigação do grupamento "Síndrome da morte súbita na infância", assim como dos fatores maternos, afecções respiratórias perinatais, prematuridade, asfixia/hipóxia, desnutrição e anemias nutricionais e diminuição percentual para as 
malformações congênitas, causas externas e infecções perinatais. Também houve redução nas causas indeterminadas e inespecíficas, aqui denominadas "outras causas".

\section{Tabela 4}

Concordância observada e índice Kappa entre a causa básica dos óbitos infantis investigados (DO original e após investigação) segundo categorias da lista reduzida de tabulação de causas de mortalidade infantil. Belo Horizonte, 2010 e 2011.

\begin{tabular}{|c|c|c|c|c|c|c|c|}
\hline \multirow[t]{3}{*}{ LIR-MI* } & \multicolumn{4}{|c|}{ Causa Básica } & \multirow[t]{3}{*}{$\mathrm{CO}$} & \multirow[t]{3}{*}{ Kappa } & \multirow[t]{3}{*}{ IC95\% } \\
\hline & \multicolumn{2}{|c|}{$\begin{array}{l}\text { DO } \\
\text { original }\end{array}$} & \multicolumn{2}{|c|}{$\begin{array}{l}\text { DO após } \\
\text { investigação }\end{array}$} & & & \\
\hline & $\mathrm{n}$ & $\%$ & $\mathrm{n}$ & $\%$ & & & \\
\hline $\begin{array}{l}\text { Fatores maternos (P00; P01.0-P01.6; } \\
\text { P01.8-P01.9; P02.2-P02.3; P02.7-P02.9; }\end{array}$ & \multicolumn{6}{|c|}{ Fatores maternos (P00; P01.0-P01.6; } & \\
\hline P04; P96.4; P05) & 2 & 1,3 & 18 & 12,1 & 0,88 & 0,08 & $-0,091-0,246$ \\
\hline \multicolumn{8}{|l|}{ Prematuridade (P07; P22.0; P25-P26; } \\
\hline P27.1; P28.0; P52; P77) & 5 & 3,4 & 8 & 5,4 & 0,97 & 0,6 & $0,279-0,918$ \\
\hline $\begin{array}{l}\text { Asfixia/Hipóxia (P20-P21; P03; P10-P15; } \\
\text { P24.0-P24.2; P24.4-P24-9; P01.7; P02.2; }\end{array}$ & & & & & & & \\
\hline $\mathrm{P} 02.1 ; \mathrm{P} 02.4-\mathrm{P} 02.6)$ & 29 & 19,5 & 36 & 24,2 & 0,89 & 0,67 & $0,521-0,812$ \\
\hline $\begin{array}{l}\text { Infecções perinatais (P36; P23; P35.1- } \\
\text { P35.2; P35.4-P35.9; P37-P39; A50; B20- }\end{array}$ & \multicolumn{6}{|c|}{ Infecções perinatais (P36; P23; P35.1- } & \\
\hline$B-24)$ & 22 & 14,8 & 17 & 11,4 & 0,87 & 0,44 & $0,231-0,650$ \\
\hline \multicolumn{8}{|l|}{ Afecções respiratórias perinatais (P28.1- } \\
\hline P28.4; P28.8-P28.9; P22.1) & 1 & 0,7 & 2 & 1,3 & 0,98 & $-0,01$ & $-0,022-0,004$ \\
\hline $\begin{array}{l}\text { Transtornos cardiovasculares origina- } \\
\text { dos no período perinatal (P29) }\end{array}$ & \multicolumn{6}{|c|}{ Transtornos cardiovasculares origina- } & $-0016-0,003$ \\
\hline \multicolumn{8}{|l|}{ Infecções da criança (J12-J18; J00-J06; } \\
\hline \multicolumn{8}{|l|}{ J09-J11; J20; J21; J22; A00-A09; E86- } \\
\hline E87; A39; A40-A41; G00; G03-G04) & 16 & 10,7 & 18 & 12,1 & 0,89 & 0,47 & $0,248-0,690$ \\
\hline \multicolumn{8}{|l|}{ Desnutrição e anemias nutricionais } \\
\hline (D-50-D53; E40-E64) & 0 & 0,0 & 1 & 0,7 & 0,99 & 0 & $0,0-0,0$ \\
\hline Asma (J45-J46) & 0 & 0,0 & 2 & 1,3 & 0,99 & 0 & $0,0-0,0$ \\
\hline \multicolumn{8}{|l|}{ Causas externas nas crianças (J69; P24.3; } \\
\hline W78; W79-W80; W84; V01-Y89) & 36 & 24,2 & 15 & 10,1 & 0,86 & 0,41 & $0,211-0,615$ \\
\hline $\begin{array}{l}\text { Malformações congênitas (Q.20-Q28; } \\
\text { Q00-Q07; Q42; Q35-Q37; Q39; Q79.0- }\end{array}$ & & & & & & & \\
\hline Q79.5; Q40.1; Q60.0-Q60-6; Q90; Q00-Q99) & 4 & 2,7 & 1 & 0,7 & 0,97 & $-0,01$ & $-0,028-0,006$ \\
\hline \multicolumn{8}{|l|}{ Doenças imunizáveis (A15-A19; A33-A35; } \\
\hline G00.0; P35.0; P35.3) & 1 & 0,7 & 1 & 0,7 & 1 & 1 & $1,0-1,0$ \\
\hline \multicolumn{8}{|l|}{ Síndrome da morte súbita na infância } \\
\hline (R95) & 1 & 0,7 & 10 & 6,7 & 0,93 & $-0,01$ & $-0,035-0,010$ \\
\hline Outras causas & 31 & 20,8 & 19 & 12,8 & 0,81 & 0,33 & $0,146-0,524$ \\
\hline Total & 149 & & 149 & & 0,47 & 0,38 & $0,192-0,576$ \\
\hline
\end{tabular}

Fonte: SIM e CPOMFI - Secretaria Municipal de Saúde de Belo Horizonte.

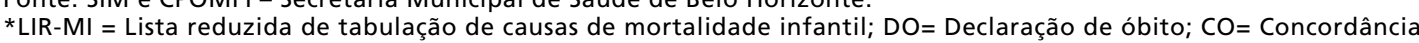
observada. 


\section{Discussão}

A concordância entre a causa básica de óbito da DO original e da DO após investigação dos óbitos infantis evitáveis investigados pelo CMPOFI em Belo Horizonte, em 2010-2011, foi avaliada como fraca neste estudo. Aproximadamente metade das $\mathrm{CB}$ de óbito da DO original não foram concordantes com as CB após investigação. Ou seja, neste estudo verificou-se que, apesar do grande percentual de óbitos ocorrido em ambiente hospitalar $(75,8 \%)$, a DO não foi adequadamente preenchida no que diz respeito às causas do óbito.

Estudos brasileiros que compararam concordância entre a DO original e a DO após investigação de óbitos infantis também apresentaram, de forma geral, baixa concordância.8,17,18 Em 1999, Lansky et al.10 avaliaram a confiabilidade da causa básica de óbito perinatal em Belo Horizonte e também verificaram baixa concordância da causa básica entre a DO original e a DO após investigação em prontuário hospitalar.

A qualificação da causa básica de óbito é um dos propósitos dos comitês de investigação de óbitos fetal e infantil, uma vez que ainda persistem problemas quanto à fidedignidade da informação sobre a causa da morte declarada pelo médico no atestado de óbito. ${ }^{7}$ A Secretaria Municipal de Saúde de BH (SMSA), o Ministério da Saúde e o Conselho Federal de Medicina (CFM) divulgam sistematicamente informações e capacitam os médicos para o adequado preenchimento da DO. 9 O produto da investigação de óbitos, inclusive a DO após investigação proposta pelo CMPOFI, é repassado à gestão do SIM a fim de possibilitar a correção, quando indicada, da causa básica e de outras variáveis da DO e assim agregar valor a esse banco. ${ }^{11} \mathrm{Tal}$ fato aponta a importância dos Comitês para o aprimoramento das análises da mortalidade infantil.

Os óbitos evitáveis estudados apresentaram um perfil diferente do grupo de óbitos infantis do município de Belo Horizonte. Conforme esperado e de acordo com os critérios para investigação de óbitos infantis da SMSA-BH, verificou-se no grupamento de óbitos evitáveis o predomínio do componente pós-neonatal, de crianças com peso ao nascer maior que $1500 \mathrm{~g}$ e de termo. Foram 149 óbitos de crianças com grande chance de sobrevivência, que representavam $22,4 \%$ dos óbitos infantis ocorridos no período e que morreram devido a problemas no acesso, na assistência de qualidade à saúde ou na insuficiência de políticas públicas intersetoriais. 19,20 Houve mudança importante no perfil de mortalidade por grupo de causa entre a DO original e a DO após investigação, o que possibilitou maior visibilidade para dois grupos da LIR-MI, 16 o de fatores maternos e da SMSI.

A asfixia/hipóxia foi o principal grupo de causa de óbito após a investigação, com índice Kappa fraco para esse grupo. Os óbitos por asfixia/hipóxia são evitáveis e refletem a qualidade da assistência ao parto e ao recém-nascido,19-21 sinalizando os serviços que não estão preparados para uma boa atenção perinatal. 10 Para reduzir a morbi-mortalidade por asfixia/hipóxia é preciso garantir o acesso a serviços de qualidade em tempo oportuno, evitar as transferências de gestantes em trabalho de parto, qualificar a assistência ao parto com a implantação das boas práticas obstétricas baseadas em evidências científicas, manter recursos materiais suficientes e equipe multiprofissional capacitada para identificar riscos e prestar atendimento de qualidade à gestante e ao recém-nascido. $2,3,22$

Constatou-se que os fatores maternos se apresentaram em maior frequência na DO após investigação, uma vez que causas pouco específicas como afecções originadas no período perinatal passaram a ser classificadas neste grupo. Em Recife, Sarinho et al. 18 detectaram situação similar, com aumento de $365,2 \%$ nas causas maternas gestacionais após revisão de prontuários, e esta foi a principal causa de óbito neonatal. O elevado percentual de fatores maternos apontados como causa básica de óbito na DO após investigação remete à necessidade de garantia do diagnóstico precoce, em especial o tratamento e acompanhamento das doenças infecciosas, da hipertensão e de agravos como a ruptura prematura da placenta. 23

Em relação ao grupamento prematuridade, o índice Kappa foi intermediário quando se comparou a DO original e a DO após investigação. Dois óbitos pós-neonatais domiciliares classificados pela DO original como causa externa passaram para esse grupo, assim como outros dois óbitos neonatais precoces, que tiveram como causa básica septicemia não especificada. São óbitos potencialmente evitáveis que necessitam ter seus determinantes identificados a fim de que sejam propostas medidas eficientes para sua prevenção. ${ }^{24} \mathrm{Um}$ caso identificado como prematuridade iatrogênica (cesariana eletiva sem indicação) aponta a relevância do problema, relacionado às elevadas taxas de cesarianas existentes no país. $6,22,24$

A concordância da causa básica no grupo das infecções da criança foi intermediária. Seis casos inicialmente classificados como causa externa passaram a compor este grupo sendo que um deles ocorreu no domić́lio e dois em um serviço de 
urgência, apontando a dificuldade de reconhecimento de risco e de acesso oportuno ao serviço de saúde. Este grupo é representado basicamente pelas diarréias infecciosas e pneumonia. Apesar da extensa rede assistencial disponível no município ainda ocorrem óbitos por estas causas evitáveis de morte, indicando a necessidade de se estabelecer políticas públicas intersetoriais capazes de detectar precocemente famílias em situação de vulnerabilidade, ${ }^{5}$ pela intrínseca relação entre os determinantes sociais e essas causas de morte em crianças. Promover equidade é uma forma de enfrentar os problemas relativos às infecções da criança uma vez que é fundamental garantir acesso aos serviços e manter qualidade na assistência, reconhecendo as diferenças e promovendo tratamento diferente a estes. 25

No presente estudo, a Síndrome da Morte Súbita da Infância (SMSI) foi a sexta principal causa de morte após investigação, indicando a importância da investigação dos óbitos para sua definição como causa básica. Em países desenvolvidos a SMSI tem grande importância como causa de óbito infantil,26 uma vez que as mortes por causas evitáveis já foram minimizadas. Nos países em desenvolvimento sua incidência é pouco conhecida. 27 No Brasil a SMSI é subdiagnosticada e são poucas as informações divulgadas sobre os fatores de riscos e formas de prevenção. 26,27 Neste estudo, dos dez casos de SMSI identificados após a investigação, nove ocorreram fora do ambiente hospitalar e sete apresentavam DO original de causa externa. Em todos foi realizada a necropsia pelo Instituto de Medicina Legal (IML) que não realiza exame anatomopatológico para causas não externas como um serviço de verificação de óbitos (SVO). Sabe-se que para detectar possíveis casos de SMSI é necessária a investigação adequada dos óbitos, 26,27 capacitação dos médicos para sua identificação e realização de autopsia por SVO26,27 não disponível em Minas Gerais. Neste estudo os casos classificados como SMSI não passaram por investigação minuciosa com exame post mortem completo e exame do local onde ocorreu o óbito conforme preconizado por Willinger et al.28 Por outro lado segundo Bergman, ${ }^{29}$ a SMSI pode ser diagnosticada mesmo quando não realizada a autopsia, conforme procedimento deste estudo.

O grupo das causas externas da LIR-MI apresentou concordância intermediária, com diminuição no número de casos após a investigação. Entretanto, apesar do número menor, os casos infantis de morte violenta devem ser considerados como um problema de saúde pública, que deve ser enfrentado em conjunto com outras políticas públicas, considerando a relação entre violência e desigualdade social. 6
Um aspecto a ser ressaltado foi o fato da investigação dos óbitos possibilitar que 20 DO originalmente classificadas como "outras causas" por apresentarem causa pouco específica ou mal definida fossem classificadas após investigação em algum dos 13 grupos definidos de causas da LIR-MI, melhorando a qualidade da informação com o reconhecimento destes óbitos como evitáveis. ${ }^{7,11}$

Dentre os óbitos estudados foi identificado um óbito por kernicterus, um por leishmaniose visceral e um por varicela, importantes eventos sentinelas 30 para o sistema de saúde. O primeiro indica falha na assistência ao RN, o segundo indica falha nas ações intersetoriais de vigilância à saúde 5 ; o terceiro aponta falha na prevenção de uma doença imunizável. Além desses, 8,7\% $(\mathrm{n}=13)$ dos óbitos ocorreram fora do ambiente hospitalar e merecem também destaque como eventos sentinelas 30 especiais, uma vez que assinalam dificuldade de acesso ao serviço de saúde seja por dificuldade da família em reconhecer o risco, ou por problemas relacionados aos processos assistenciais ou falha na instituição do tratamento.

Uma limitação deste estudo refere-se ao fato de poder ter ocorrido subestimação dos fatores maternos e da prematuridade como causas de óbito potencialmente evitáveis, uma vez que foram analisados somente os óbitos de crianças com peso ao nascer maior que $1500 \mathrm{~g}$ e sem malformação congênita grave (MFC). Pode também ter ocorrido superestimação da SMSI devido a não realização de autópsias.

Este estudo possibilitou a identificação dos óbitos infantis por causas evitáveis, apontando problemas no acesso e/ou na qualidade da assistência no pré-natal, parto e à criança. Além disso, identificouse a baixa qualidade da informação sobre a causa básica dos óbitos infantis notificados inicialmente ao SIM por meio da DO e a necessidade de se investir na constituição de comitês de prevenção de óbito para qualificar a informação e a análise das circunstâncias dos óbitos.

Capacitar os médicos para o adequado preenchimento da DO, e a instituição do Serviço de Verificação de Óbito são estratégias fundamentais para qualificar as informações sobre as causas do óbito infantil. Qualificar a informação é o primeiro passo para avaliação adequada do perfil da mortalidade infantil por causas e seus fatores determinantes. Assim poderemos avançar subsidiando de forma adequada a tomada de decisão pelos gestores com o propósito de melhorar a assistência e reduzir a mortalidade infantil. 


\section{Referências}

1. França E, Lansky S. Mortalidade infantil neonatal no Brasil: situação, tendências e perspectivas. In: Rede Interagencial de Informações para a Saúde (RIPSA). Demografia e saúde: contribuição para análise de situação e tendências. Brasília: Organização Pan-Americana da Saúde; 2009 .

2. Lansky S, França E, Leal MC. Mortes perinatais evitáveis em Belo Horizonte. Minas Gerais. 1999. Cad Saúde Pública 2002; 18 (5): 1389-400.

3. Lansky S, França E, César CC, Neto LCM, Leal MC. Mortes perinatais e avaliação da assistência ao parto em maternidades do Sistema Único de Saúde em Belo Horizonte, Minas Gerais, Brasil, 1999. Cad Saúde Pública 2006; 22 (1): 117-30

4. Brasil. Ministério do Planejamento, Orçamento e Gestão (Brasil). Objetivos de Desenvolvimento do Milênio, Relatório Nacional de Acompanhamento. IPEA 2014; 6675 .

5. Jones G, Stekette RW, Black RE, Bhutta ZA, Morris SS How many child deaths can we prevent this year? Lancet 2003; 362 (9377): 65-71.

6. Observatório do Milênio de Belo Horizonte. Relatório de acompanhamento dos Objetivos de desenvolvimento do Milênio Belo Horizonte 2014. 1 ed. Belo Horizonte; 2015.

7. Laurenti R, Mello Jorge MHP, Gotlieb SLD. Mortalidade segundo causas: considerações sobre a fidedignidade dos dados. Rev Panan Salud Publica 2008; 23 (5); 349-56.

8. Mendonça EF, Goulart EMA, Machado JAD Confiabilidade da declaração de causa básica de mortes infantis em região metropolitana do sudeste do Brasil. Rev Saúde Pública 1994; 28 (5): 385-91

9. Drumond EF, Ishitani LH, Machado CJ. Óbitos neonatais precoces em Belo Horizonte: um enfoque de causas múltiplas. Cad Saúde Colet. 2010; 18 (3): 380-4.

10. Lansky S, França E, Ishitani L, Xavier CC. Confiabilidade da informação sobre o óbito perinatal em Belo Horizonte, 1999: causas de óbito e variáveis selecionadas. Cad Saúde Colet. 2010; 18 (1): 63-70.

11. Mello Jorge MHP, Laurenti R, Gotlieb SLD. Avaliação dos sistemas de informação em saúde no Brasil. Cad. Saúde Colet. 2010; 18 (1): 7-18.

12. Brasil. Ministério da Saúde. Secretaria de Vigilância em Saúde. Secretaria de Atenção à Saúde. Manual de vigilância do óbito infantil e fetal e do Comitê de Prevenção do Óbito Infantil e Fetal. Brasília, DF; 2009.

13. Brasil. Ministério da Saúde. Secretaria de Vigilância em Saúde. Departamento de Análise de Situação em Saúde. Manual de Instruções para o preenchimento da declaração de óbitos. 4 ed. Brasília, DF; 2011.

14. OMS (Organização Mundial da Saúde). Classificação Estatística Internacional de Doenças e Problemas Relacionados à Saúde. $10^{\circ}$ Revisão. São Paulo: Centro Brasileiro de Classificação de Doenças; (vol. 1 e 2), 1995.

15. Fleiss JL: Statical Methods for Rates and Proportions, 2 ed New York: John Wiley \& Sons; 1981.
16. Brasil. Ministério da Saúde. Secretaria de Vigilância em Saúde. Saúde Brasil 2011: uma análise da situação de saúde e a vigilância da saúde da mulher. Brasília, DF; 2012. p. $163-82$

17. Pedrosa LDCO, Sarinho SW, Ordonha MR. Análise da qualidade da informação sobre causa básica de óbitos neonatais registrados no Sistema de Informações sobre Mortalidade: um estudo para Maceió, Alagoas, Brasil, 2001-2002. Cad Saúde Pública 2007; 23 (10): 2385-95.

18. Sarinho SW, Coutinho SB, Acioli TML, Menezes TF, Abreu MAP, Lacerda N, Guimarães MJB. Mortalidade neonatal em Recife, PE: causas básicas e grau do conhecimento dos neonatologistas acerca do preenchimento das declarações de óbito. Pediatria (São Paulo) 2001; 23 (4): 279-84

19. Malta DC, Duarte EC, Escalante JJC, Almeida MF, Sardinha LMV, Macário EM, Monteiro RA, Neto OLM. Mortes evitáveis em menores de um ano, Brasil, 1997 a 2006: contribuição para a avaliação de desempenho de Sistema Único de Saúde. Cad Saúde Pública. 2010; 26(3): 481-91.

20. Malta DC, Sardinha L, Moura L, Lansky S, Leal MC, Szwartwald CL, França E, Almeida MF, Duarte EC. Atualização da lista de causas de mortes evitáveis por intervenções do Sistema Único de Saúde do Brasil. Epidemiol Serv Saúde. 2010; 19: 173-6.

21. Daripa M, Caldas HMG, Flores LPO, Waldvogel BC, Guinsburg R, Almeida MFB. Asfixia perinatal associada à mortalidade neonatal precoce: estudo populacional dos óbitos evitáveis. Rev Paul Pediatr. 2013; 31 (1): 37-45.

22. Lansky S, Friche MAL, Silva AAM, Campos D, Bittencourt SDA, Carvalho ML, Frias PG, Cavalcante RS, Cunha AJLA. Pesquisa Nascer no Brasil: perfil da mortalidade neonatal e avaliação da assistência à gestante e ao recém nascido. Cad Saúde Pública. 2014; 30 (Supl.): S1-15.

23. Domingues RMSM, Hartz ZMA, Dias MAB, Leal MC. Avaliação da adequação da assistência pré-natal na rede SUS do município do Rio de Janeiro, Brasil. Cad Saúde Pública. 2012; 28 (3): 425-37.

24. Barros FC, Victora CG, Barros AJ, Santos IS, Albernaz E, Matijasevich A, Domingues MR, Iclowitz IKT, Hallal PC, Silveira MF, Vaughan JP. The challenge of reducing neonatal mortality in middle-income countries: findings from three Brazilian birth cohorts in 1982, 1993, and 2004. Lancet. 2005; 365 (9462): 847-54.

25. Campos GWS. Reflexões temáticas sobre equidade e saúde: o caso do SUS. Saúde Soc. 2006; 15 (2): 23-33.

26. CastroEC, Peres LC. Síndrome da morte súbita na infância. Medicina (Ribeirão Preto) 1998; 31: 584-94.

27. Nunes ML, Pinho APS, Aerst D, Sant'Anna A, Martins MP, Costa JC. Síndrome da morte súbita do lactente: aspectos clínicos de uma doença subdiagnosticada. J Pediatr (Rio J.). 2001; 77 (1): 29-34.

28. Willinger M, James LS, Catz C. Defining the sudden infant death syndrome (SIDS): deliberations of an expert panel convened by the National Institute of Child Health an Human Development. Pediatr Pathol. 1991; 11: 677-84. 
29. Bergman AB. Studying sudden infant death syndrome in developing country. J Pediatr (Rio J.). 2006; 82: 4-5.

Recebido em 17 de julho de 2015

Versão final apresentada em 28 de setembro de 2015

Aprovado em 2 de outubro de 2015
30. Rutstein DD, Berenberg W, Chalmers TC, Child CG, Fishman AP, Perrin EB. Measuring the quality of medical care: a clinical method. New Engl J Med. 1976; 294 (11): 582-8. 